\title{
Problemática de las pirolusitas de Ímini en la fabricación de ferromanganesos para aplicaciones metalúrgicas
}

\author{
I. Gómez-Pinilla ${ }^{(*)}$, M.T. Larrea ${ }^{(* *)}$ y F.J. Valle ${ }^{(* * *)}$
}

\begin{abstract}
Resumen Se estudia la posibilidad de eliminar la impureza de plomo de la pirolusita de Ímini (Marruecos). El material se somete a un proceso mineralúrgico que permite separar seis fracciones basándose en su diferente densidad y comportamiento electromagnético. De las quince especies mineralógicas encontradas, ninguna incluye el plomo en su formulación teórica. Para detectar la presencia de este elemento se ha recurrido al microanálisis por dispersión de energías (EDX) realizando un mapping de plomo en cada una de las fracciones. Este elemento se concentra en los cristales de psilomelana. La composición química de la psilomelana se ha comparado con la de una hollandita recogida de la bibliografía. La presencia del plomo dentro de la red de la psilomelana impide su separación por procedimientos mineralúrgicos convencionales y, por tanto, hace inviable la utilización de este material en la producción de ferromanganeso.
\end{abstract}

Palabras clave: Pirolusita. Plomo. Metalurgia. Microscopía electrónica de barrido. Microanálisis por dispersión de energías.

\section{Problematic of the Ímini pyrolusites in the production of ferromanganese for metallurgical applications}

\begin{abstract}
The possibility of eliminating lead impurities from the pyrolusite of Ímini (Morocco) has been studied. The material undergoes a minerallurgical process which allows the separation of six fractions based on their differences in density and electromagnetic behaviour. Among the fifteen mineralogical species found, none of them contains lead in its theorical formulation. In order to detect the presence of this element the microanalysis by dispersive energies was used and a "mapping" of lead was made in each of the fractions. This element is concentrated in the psylomelane crystals. The chemical composition of the psylomelane was compared with that of the hollandite, taken from the references. The presence of lead inside the psylomelane crystalline structure avoids its separation by a classical minerallurgic of process and therefore the use of this material is not feasible for the production of ferromanganese.
\end{abstract}

Keywords: Pirolusite. Lead. Metallurgy. Scanning electronic microscopy. Dispersive energy microanalysis.

\section{INTRODUCCIÓN}

Aunque habitualmente se designa al $\mathrm{MnO}_{2}$ como pirolusita, existen diversos óxidos de $\mathrm{Mn}(\mathrm{IV})$ en la naturaleza que tienen nombres diferentes y

(*) Centro de Espectrometría Atómica. Univ. Complutense de Madrid. Ciudad Universitaria. 28040-Madrid (España).

(**) Centro Nacional de Investigaciones Metalúrgicas, CENIM (CSIC), Avda. de Gregorio del Amo, 8. 28040Madrid (España).

(***) Instituto de Cerámica y Vidrio. (CSIC). Arganda del Rey. 28500-Madrid (España). que pertenecen a algunos de estos tres grupos: Óxidos normales o hidratados, óxidos con presencia de concentraciones apreciables de $\mathrm{Mn}$ (II) y óxidos impurificados con otros elementos.

Entre los del primer grupo está la pirolusita (tetragonal) y la ramsdellita (ortorrómbico), ambas $\mathrm{MnO}_{2}$; entre los del segundo, se encuentran la vernadita (tetragonal) $\mathrm{Mn}(\mathrm{OH})_{4}$, la bixbyita (cúbico) $\mathrm{MnO}_{2} \cdot \mathrm{MnO}$ y la hausmanita (tetragonal) $\mathrm{Mn}_{3} \mathrm{O}_{4}$; el tercer grupo lo constituyen la janggunita (ortorómbico) $\mathrm{MnO}_{2}\left(\mathrm{Mn}^{+2}, \mathrm{Fe}^{+2}\right) \mathrm{O}_{8}(\mathrm{OH})_{6}, 1 \mathrm{a}$ 
criptomelana (monoclínico) $\mathrm{KMn}_{8} \mathrm{O}_{16}$ y la psilomelana (monoclínico) $4 \mathrm{MnO}_{2} \cdot 2(\mathrm{Mn}, \mathrm{Ba}, \mathrm{Ca}, \mathrm{O})$.

La pirolusita procedente de Ímini (Marruecos) contiene óxidos de dos de los tres grupos citados en su composición. En este yacimiento, el mineral se presenta asociado en capas con dolomías del Cenomaniense. El horizonte mineral está formado por capas elipsoidales de hasta $19 \mathrm{~km}$ de desarrollo con áreas explotables entre 150 y $400 \mathrm{~m}$ de anchura (1).

La composición química y mineralógica de este material ha sido estudiada por P. Ortega et al. (2), utilizando las técnicas de espectrometría de emisión óptica con plasma de acoplamiento inductivo (ICPOES) y microsonda electrónica (EPMA); en las tablas I y II se ofrecen los resultados que encuentran estos autores.

Se tiene constancia de la utilización de pirolusita en la industria del hierro desde los egipcios; no obstante, su aplicación formal en la fabricación de ferroaleaciones y aceros data de comienzos del siglo XIX (3).

Los materiales pirolusíticos tienen aplicaciones en las siguientes áreas: a) Metalurgia, obtención de ferromanganesos. b) Industria química, fabricación de sales de manganeso y c) Cerámica, coloración de ladrillos, eliminación del "corazón negro", etc. (4 y 5).

La principal aplicación metalúrgica de la pirolusita reside en la obtención de ferromanganesos. Esta

TABla I.- Composición química de un material pirolusítico obtenida por espectrometría de emisión óptica con plasma de acoplamiento inductivo ICP-OES

TABLE I.- Chemical composition of pyrolusite material carried out by inductively coupled plasma optical emission spectrometry

\begin{tabular}{|c|c|c|}
\hline Elemento & $\begin{array}{c}\text { Conc. de } \\
\text { elemento }\end{array}$ & $\begin{array}{c}\text { Conc. en } \\
\text { óxido }\end{array}$ \\
\hline $\mathrm{Si}$ & $2,53 \%$ & $5,42 \%$ \\
$\mathrm{Al}$ & & $1,73 \%$ \\
$\mathrm{Fe}$ & & $0,86 \%$ \\
$\mathrm{Ca}$ & & $2,76 \%$ \\
$\mathrm{Mg}$ & & $1,97 \%$ \\
$\mathrm{Ba}$ & $0,57 \%$ & $0,64 \%$ \\
$\mathrm{~K}$ & $0,27 \%$ & $0,33 \%$ \\
$\mathrm{~Pb}$ & $0,31 \%$ & $0,33 \%$ \\
$\mathrm{Cu}$ & $0,14 \%$ & $0,18 \%$ \\
$\mathrm{Na}$ & no detec. & \\
$\mathrm{Ti}$ & $360 \mathrm{ppm}$ & \\
$\mathrm{V}$ & $200 \mathrm{ppm}$ & \\
$\mathrm{P}$ & $360 \mathrm{ppm}$ & \\
$\mathrm{As}$ & $200 \mathrm{ppm}$ & \\
$\mathrm{Zn}$ & $230 \mathrm{ppm}$ & \\
$\mathrm{Co}$ & $60 \mathrm{ppm}$ & \\
\hline
\end{tabular}

TABLA II.- Composición mineralógica de una material pirolusítico obtenida por microanálisis sobre una población de 100 granos

TABLE II.- Mineralogic composition of pyrolusite material carried out by microanalysis over 100 grains

\begin{tabular}{|c|c|c|c|}
\hline \multicolumn{2}{|c|}{ Fracción $>63 \mu \mathrm{m}$} & \multicolumn{2}{c|}{ Fracción 63-37 $\mu \mathrm{m}$} \\
\hline Componente & $\begin{array}{c}\mathrm{n}^{\circ} \text { de } \\
\text { granos }\end{array}$ & Componente & $\begin{array}{c}\mathrm{n}^{\circ} \mathrm{de} \\
\text { granos }\end{array}$ \\
\hline Cuarzo & 30 & Psilomelana & 13 \\
Dolomita & 21 & Rodonita & 8 \\
Apatito & 8 & Blenda & 4 \\
Moscovita & 3 & Hauerita & 2 \\
Ortosa & 3 & Magnetita & 2 \\
Magnetita & 3 & Apatito & 1 \\
Rutilo & 2 & & \\
\hline
\end{tabular}

ferroaleación se utiliza en la fabricación del acero como aleante, para eliminar el azufre y para proporcionar templabilidad al acero (6). Para esta aplicación, se requieren pirolusitas con elevado contenido de manganeso y bajo nivel de impurezas, sobre todo por lo que concierne al plomo (7). Este elemento presenta una baja solubilidad en el hierro, por lo que su existencia en esta materia prima invalida su empleo en la fabricación de ferromanganeso.

Cuando la pirolusita no puede emplearse en la fabricación de ferromanganeso por el motivo expuesto, se usa entonces para aplicaciones en la industria química y cerámica.

\subsection{Objetivo}

El objetivo de este trabajo consiste en estudiar la posibilidad de utilizar en metalurgia la pirolusita procedente del yacimiento de Ímini (Marruecos). En este sentido, se verá la forma de separar el plomo. Para ello se procederá a realizar un análisis mineralúrgico que, con la separación y concentración de los distintos minerales presentes en el material, facilite la localización de este elemento como paso previo a su separación.

\section{PARTE EXPERIMENTAL}

\subsection{Equipamiento}

Difractómetro de rayos X (DRX) Philips modelo MPD 1880 con las siguientes características: Tubo de rayos $\mathrm{X}$ con ánodo de cobre. Radiación $\mathrm{Cu}$, $\mathrm{K}_{\alpha}=0,154052 \mathrm{~nm}$, potencia de tubo $2,2 \mathrm{~kW}$. Goniómetro vertical provisto de una rendija de divergencia automática. Monocromador de grafito. 
Detector proporcional. Ordenador IBM modelo System 2555 X-GL y Software PC-APP.

Lupa binocular (BH) SZ-III de la casa Olimpus, provista de zoom que permite hasta 40 aumentos y un tubo de observación binocular.

Microscopio electrónico de barrido digitalizado Zeiss DSM-950 con sistema de microanálisis de rayos X por dispersión de energías (EDX) Tracor Northem ZX-II.

Espectrómetro de emisión óptica con fuente de plasma de acoplamiento inductivo (ICP-OES) Jarrell-Ash modelo ICAP-61, con frecuencia de oscilador de $27,7 \mathrm{MHz}$, policromador con 28 tubos fotomultiplicadores, red de difracción de 1,510 trazos $\mathrm{mm}^{-1}$ con montaje Pachen-Runge, resolución $0,031 \mathrm{~nm}$ en $1^{\mathrm{er}}$ orden y $0,015 \mathrm{~nm}$ en $2^{\circ}$ orden.

\subsection{Procedimiento}

- La separación mineralúrgica se ha llevado a cabo sobre el todo-uno de la muestra, para facilitar su posterior caracterización mineralógica. Se ha sometido la muestra a un proceso de separación electromagnética y por líquidos densos, cuyo esquema se recoge en la figura 1 .

- El análisis por DRX se ha efectuado por el método de polvo. La muestra se ha molido en mortero de ágata hasta un tamaño de partícula inferior a $35 \mu \mathrm{m}$. Se ha ampliado la escala del difractograma en X5 para detectar las impurezas en vista de la intensidad de pico del componente mayoritario, pirolusita, que impide detectar los picos de las impurezas.

- En SEM-EDX, las muestras procedentes de las seis fracciones a analizar con contenidos que no han superado los 200 granos por fracción se han colocado extendidas sobre portamuestras que poseen un papel doblemente adhesivo, lo que

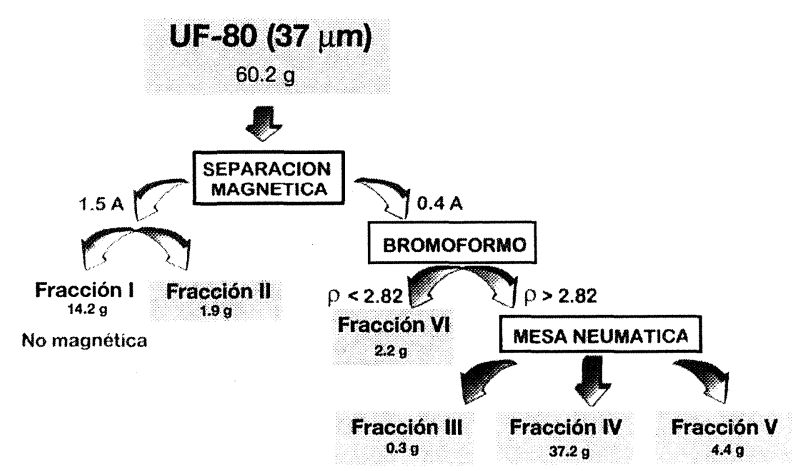

Fig. 1.- Diagrama de separación mineralúrgica del material UF-80.

FIG. 1.-Diagram of minerallurgical separation of UF-80 material. permite que los cristales queden fijos. Para su observación y consideración microanalítica en el microscopio electrónico se han metalizado con una fina capa de carbono de aproximadamente $20 \mathrm{~nm}$ de espesor. Para esta operación se ha seguido la técnica de sombreado en alto vacío $\left(5 \cdot 10^{-5}\right.$ torr $)$.

\section{RESULTADOS Y DISCUSIÓN}

Las impurezas mayoritarias que acompañan al mineral (dolomita, cuarzo, caolinita, psilomelana y criptomelana), se han identificado por DRX (Fig. 2). Para conocer y evaluar las impurezas totales (mayoritarias y minoritarias), se ha procedido a realizar una separación mineralúrgica, según el esquema ofrecido en la figura $1, \mathrm{y}$ un análisis mineralógico semicuantitativo por recuento de granos mediante EDX de las seis fracciones separadas que se ofrece en la tabla III.

Al cotejar el análisis químico expuesto en la tabla I con las especies mineralógicas recogidas en la tabla III, no ha aparecido ningún mineral al que pueda ser atribuida la presencia del plomo. No queda otra alternativa que suponer que el citado elemento entra a formar parte de la composición de alguno de los minerales presentes, incluyendo la propia pirolusita.

Muestras de las seis fracciones fueron observadas de nuevo por microscopía electrónica de barrido. Para detectar la existencia de plomo se procedió a hacer un mapping de este elemento en todas ellas, localizándose en las fracciones IV y $\mathrm{V}$ fundamentalmente y precisamente dentro de los cristales de psilomelana. Una micrografía de esta fracción $\mathrm{V}$ y el espectro de rayos $\mathrm{X}$ correspondiente a uno de los cristales más significativos de la misma se observa en la figura 3.

La presencia del pico de potasio en el espectro está justificada porque el área excitada por el haz

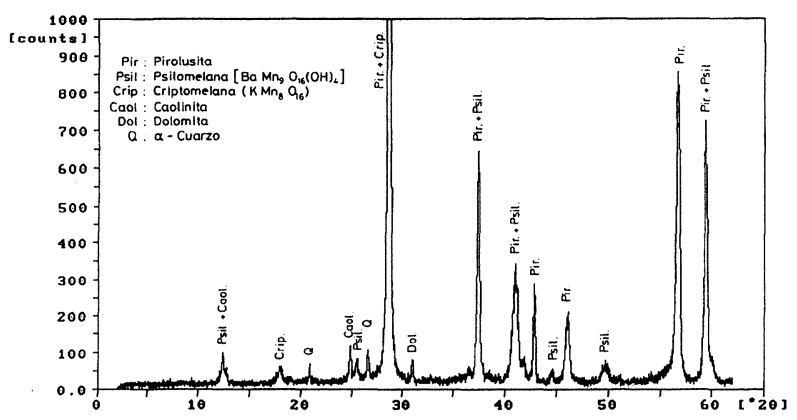

FIG. 2.- Difractograma correspondiente al material pirolusítico de Ímini UF-80.

FIG. 2.-Difractogram corresponding to the pyrolusite UF-80 material. 
TABLA III.- Composición mineralógica del material UF-80

TABLE III.- Mineralogic composition of UF-80 material

\begin{tabular}{|l|l|l|l|l|l|}
\hline Fracción I & Fracción II & Fracción III & Fracción IV & Fracción V & Fracción VI \\
\hline Dolomita +++ & Cuarzo +++ & Pirolusita +++ & Psilomenana +++ & Psilomenana +++ & Rodonita/pirolusita + \\
Cuarzo + & Dolomita ++ & Magnetita + & Criptomelana ++ & Criptomelana + & Caolinita/pirolusita ++ \\
Caolinita + & Apatito + & Calcopirita + & Pirolusita + & & Cuarzo/pirolusita +++ \\
Rodonita + & Pirolusita + & & & & Hamelita/Pirolusita +++ \\
Apatito & Cuarzo/pirolusita & & & & \\
Ortosa & & & & & \\
Rutilo & & & & & \\
Blenda & & & & & \\
\hline
\end{tabular}

$(+++)$ : mayoritario/ $(++)$ : intermedio/ (+): minoritario/
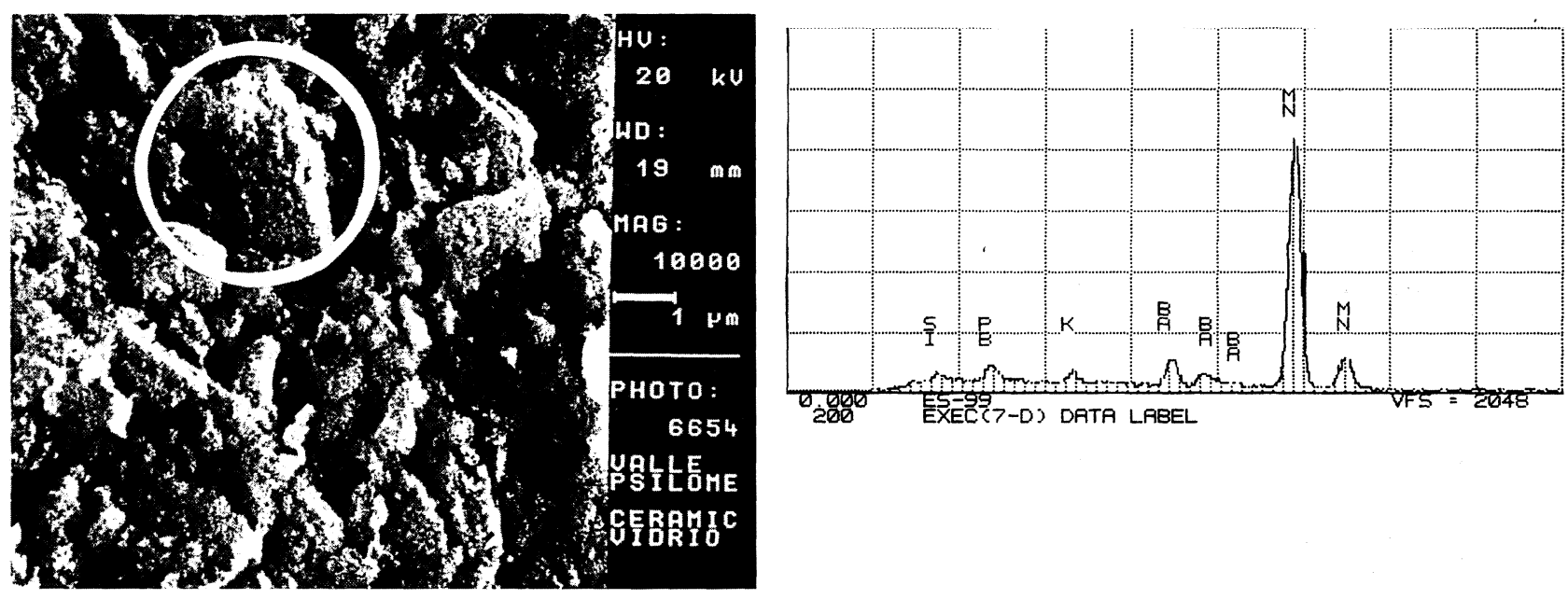

Fig. 3.- Micrografía del material pirolusítico de Ímini.

FIG. 3.- Micrograph of Imini pyrolusite material.

supere la superficie del cristal y se esté sacando información de algún cristal de criptomelana limítrofe. Por otra lado, los indicios de silicio deben atribuirse a algún cristal de cuarzo libre o mixto no detectado en el análisis por EDX, pero que se encontraba en esta fracción a pesar de no corresponderle por el proceso de separación mineralúrgica llevado a cabo.

Viendo la imposibilidad de separar la psilomelana de la criptomelana en la fracción $\mathrm{V}$ por un procedimiento mineralúrgico riguroso, como el recogido en el esquema de la figura 1, se procedió al análisis químico de la psilomelana por EDX promediando 10 granos. Un óxido mineral de $\left(\mathrm{Mn}^{4+}\right)$ de una composición química parecida es descrito como hollandita por Jeffrey et al. (8). Según estos autores, el plomo y el bario sustituyen a cationes túneles $\left(\mathrm{Mn}^{3+}\right)$ distorsionando la estructura de la red monoclínica. Los picos principales de difracción de la hollandita se ajustan a las difracciones de la psilomelana de Ímini con una ligera separación, lo que podría indicar que la psilomelana de Ímini y la hollandita (ambas cristalizan en el sistema monoclínico) deben presentar una estructura y una composición química semejante. Los resultados del análisis químico de estas dos especies mineralógicas se ofrecen en la tabla IV.

\section{CONCLUSIÓN}

Pese a que el material pirolusítico de Ímini presenta una cristalización perfecta como se aprecia en la figura 4, la imposibilidad de separar el plomo por encontrarse en la red de la psilomelana, impide su uso como materia prima en la obtención de ferromanganeso; de ahí su obligada utilización en la industria química (producción de hidroquinona, adicionándola a pinturas y barnices para favorecer el secado) y, sobre todo, en la industria cerámica y vidriera (coloración de ladrillos, decoloración de vidrios y como oxidante para evitar la formación de 
TABLA IV.- Análisis químico por EDX de la fracción V (Psilomelana) y por microsonda electrónica de la hollandita (Jeffrey E. y col. 1982)

TABLE IV.- Chemical analysis of the $V$ fraction of the psylomelane by EDX and electron microprobe of the hollandite (Jeffrey E. et al. 1982)

\begin{tabular}{|l|c|c|}
\hline & $\begin{array}{c}\text { Fracción V } \\
\text { (Psilomelana) } \\
(\%)\end{array}$ & $\begin{array}{c}\text { hollandita } \\
(\%)\end{array}$ \\
\hline $\mathrm{SiO}_{2}$ & 1,50 & 0,58 \\
$\mathrm{MnO}_{2}+\mathrm{MnO}$ & 83,70 & 67,48 \\
$\mathrm{Al}_{2} \mathrm{O}_{3}$ & - & 1,45 \\
$\mathrm{Fe}_{2} \mathrm{O}_{3}$ & - & 12,63 \\
$\mathrm{BaO}$ & 8,22 & 13,81 \\
$\mathrm{PbO}$ & 4,40 & 4,45 \\
$\mathrm{Na}_{2} \mathrm{O}$ & - & 0,58 \\
$\mathrm{~K}_{2} \mathrm{O}$ & 1,44 & - \\
\hline
\end{tabular}

"corazón negro" durante el proceso de cocción de las piezas cerámicas).

\section{REFERENCIAS}

(1) Inventario General de los Recursos de Manganeso. Ministerio de Industria y Energía. Instituto Geológico y Minero de España. Dic. 1982.

(2) Ortega, P., del Barrio, S. y Valle, F.J. Bol. Soc. Esp. Cerám. Vidrio, 34 (2), 1995: 71-75.

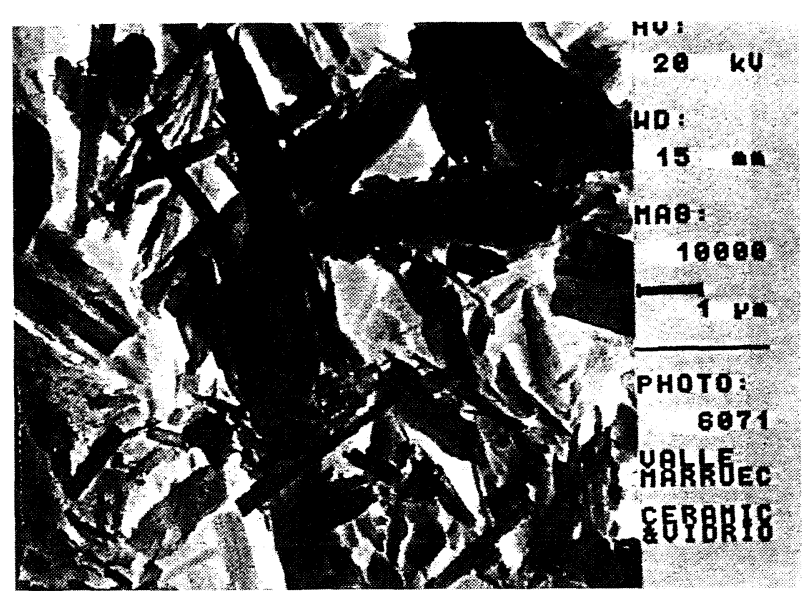

FIG. 4.- Micrografía del material pirolusítico de Ímini.

FIG. 4.- Micrograph of Imini pyrolusite material.

(3) Stanko, J.S. Manganese for Steelmakers. Council for Mineral Technology. Johannesburg, República de África del Sur, 1989.

(4) Kettani, A., Chorlet, S. y Martin, D. Qualicer. 1996: 733-738.

(5) ChORlet, S. L'Industrie Cerámique Verrière. 898, 1994: 699-703.

(6) Stanko, J.S.: Steel Makers Guide to Manganese, Mintek, Randburg 1989.

(7) ASTM-99. Standard Specification for Manganese.

(8) Jeffrey, E., Robert, B. von Dreele y Buseck, P.R. Acta Cryst. B38, 1982: 1056-1065. 